\title{
Structure of MlaFEDB lipid transporter reveals an ABC exporter fold and two bound phospholipids
}

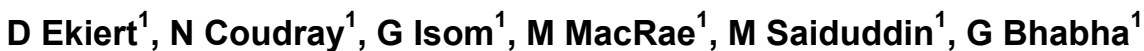 \\ ${ }^{1}$ NYU School of Medicine, New York, NY \\ dcekiert@uchicago.edu
}

In double-membraned bacteria, phospholipids must be transported across the cell envelope to maintain the outer membrane barrier, which plays a key role in antibiotic resistance and pathogen virulence. The Mla pathway has been implicated in phospholipid trafficking and outer membrane integrity, but the mechanism by which this ABC transporter facilitates phospholipid transport remains unknown. Using a combination of X-ray crystallography and cryo-EM, we have determined the structure of MlaFEDB inner membrane complex. Our structures reveal that the core transporter subunit, MlaE, adopts an $\mathrm{ABC}$ exporter fold and is related to the lipopolysaccharide export system as well as the human lipid exporter families, ABCA and ABCG. Unexpectedly, two phospholipids are bound in the outward-facing pocket of MlaFEDB, raising the possibility that multiple lipid substrates may be translocated each transport cycle. The unusual configuration of bound lipids suggests that lipid reorientation may occur before extrusion through the hydrophobic MlaD pore. Site-specific crosslinking confirms that lipids bind in this pocket in vivo. Structures of the ATPase and regulatory subunits suggest novel mechanisms for the regulation of $A B C$ transporter activity. Our work supports a model in which the Mla system may drive phospholipid export to the bacterial outer membrane, and provides insight into the function of an exporter family conserved from bacteria to humans.

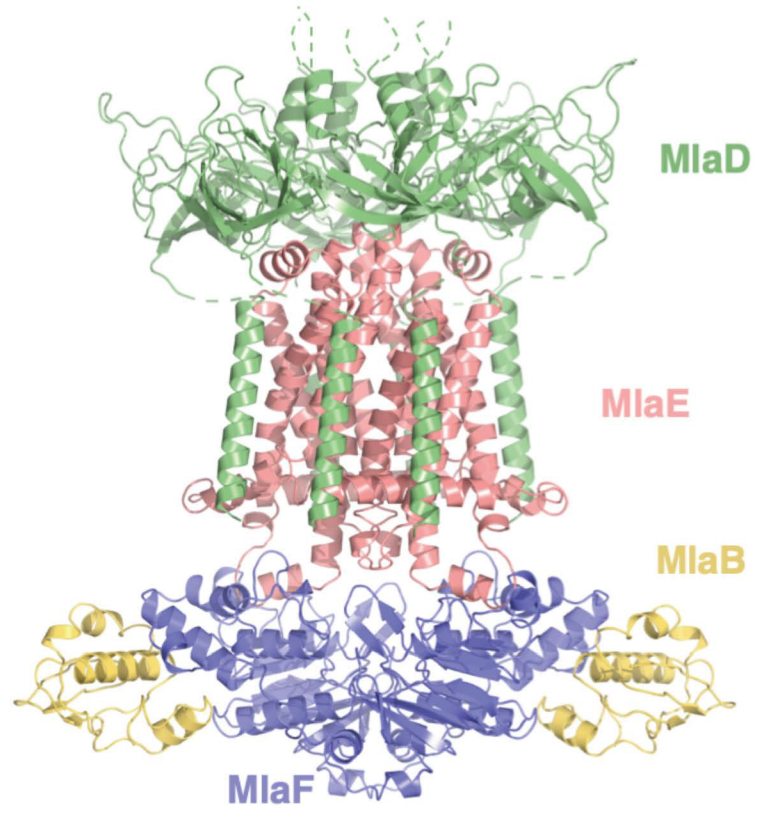

Figure 1.

Acta Cryst. (2020). A76, a82 